\title{
Removal of the Glycosylation of Prion Protein Provokes Apoptosis in SF126
}

\author{
Lan Chen ${ }^{1,3}$, Yang Yang ${ }^{1}$, Jun Han², Bao-Yun Zhang ${ }^{2}$, Lin Zhao ${ }^{1}$, Kai Nie ${ }^{2}$, Xiao-Fan Wang ${ }^{2}$, \\ Feng $\mathrm{Li}^{2}$, Chen $\mathrm{Gao}^{2}$, Xiao-Ping Dong, ${ }^{2, *}$ and $\mathrm{Cai}-\mathrm{Min} \mathrm{Xu}^{1, *}$ \\ ${ }^{1}$ National Laboratory of Medical Molecular Biology, Institute of Basic Medical Science, Chinese Academy of Medical Sciences and \\ Peking Union Medical College, Dong Dan San Tiao 5, Beijing 100005, People's Republic of China \\ ${ }^{2}$ State Key Laboratory for Infectious Disease Prevention and Control, National Institute for Viral Disease Control and Prevention, \\ Chinese Center for Disease Control and Prevention, Ying-Xin Rd 100, Beijing 100052, People's Republic of China \\ ${ }^{3}$ The State Key Laboratory of Brain and Cognitive Science, Institute of Biophysics, Chinese Academy of Sciences, \\ Chao-Yang District, Beijing 100101, People's Republic of China
}

Received 2 November 2006, Accepted 9 April 2007

\begin{abstract}
Although the function of cellular prion protein $\left(\operatorname{PrP}^{C}\right)$ and the pathogenesis of prion diseases have been widely described, the mechanisms are not fully clarified. In this study, increases of the portion of non-glycosylated prion protein deposited in the hamster brains infected with scrapie strain $263 \mathrm{~K}$ were described. To elucidate the pathological role of glycosylation profile of PrP, wild type human PrP (HuPrP) and two genetic engineering generated nonglycosylated PrP mutants (N181Q/N197Q and T183A/ T199A) were transiently expressed in human astrocytoma cell line SF126. The results revealed that expressions of non-glycosylated PrP induced significantly more apoptosis cells than that of wild type PrP. It illustrated that Bcl-2 proteins might be involved in the apoptosis pathway of non-glycosylated PrPs. Our data highlights that removal of glycosylation of prion protein provokes cells apoptosis.
\end{abstract}

Keywords: Apoptosis, Glycosylation, Neurodegenerative diseases, Pathogenesis, Prion protein

\section{Introduction}

Prion diseases or transmissible spongiform encephalopathies (TSEs) are fatal neurodegenerative disorders in humans and animals. In humans, mature $\operatorname{PrP}^{\mathrm{C}}$ is a membrane protein of 209 amino acids without N- and C-terminal signal peptides, which includes an octapeptide repeat region at N-terminal, a

\footnotetext{
*To whom correspondence should be addressed. Tel: 86-10-65296445; Fax: 86-10-65296445

E-mail: caiminxu@yahoo.com.cn (Cai-Min Xu)

Tel: 86-10-83534616; Fax: 86-10-83534616

E-mail: dongxp238@sina.com (Xiao-Ping Dong)
}

disulfide bridge between residues 179 and 214, a glycosylphosphatidylinositol anchor (GPI) at C-terminal, and two potential N-linked glycosylation sites at amino acid Asn181 and Asn197 (Prusiner, 1998).

The pathogenesis of prion diseases remains still unsettled. Accumulated data indicates that apoptosis may play a major role in TSEs and participate in the formation of spongiform tissues. Apoptosis was invariably observed in the brain tissues from the mice experimentally infected with scrapie and Creutzfeldt-Jakob disease (CJD) strains, from the cows with bovine spongiform encephalopathy (BSE) and from human cases of CJD and Fatal familial insomnia (FFI). Similar phenomena were detected in vitro, that in cell lines maintaining prion, purified abnormal isoform $\left(\mathrm{PrP}^{\mathrm{Sc}}\right)$ from mouse scrapie brain induced apoptosis in $\mathrm{N}_{2} \mathrm{~A}$ neuroblastoma cells, GT1 cells, as well as primary cerebella cultures (Cronier et al., 2004).

Recently, more and more attentions were focused on the possible neurotoxic feature of PrP. The octapeptide repeat region of $\operatorname{PrP}^{\mathrm{C}}$ was reported to play an essential role in regulating apoptosis (Nishimura et al., 2004). A synthetic peptide homologous to residues 106-126 of $\operatorname{PrP}(\operatorname{PrP} 106-126)$ maintained many characteristics of $\operatorname{PrP}^{\mathrm{Sc}}$, including the ability to induce apoptosis in neuronal cells (Ciesielski-Treska et al., 2004). The putative transmembrane domain of the human $\operatorname{PrP}^{\mathrm{C}}$, i.e., amino acids 118-135, induced oligodendrocyte (OLG) death in vitro and triggered pathways leading to neuronal apoptosis (Chabry et al., 2003). Moreover, phospholipase A activation by PrP-GPIs acted as an early event in prioninduced neurodegeneration (Bate and Williams, 2004).

Apoptosis is characterized by cell shrinkage, DNA fragmentation, protease activation, and alterations in cell surface molecules such as phosphatidylserine and the sugar residues of glycostructures. The increase in glycan exposure occurs late in the apoptotic process. However, whether the 
glycan changes on the membrane surface are the cause or the effect is largely unclear. Recent studies have shown that inhibition of N-linked glycosylation using tunicamycin (TM) induced cell apoptosis in cultured cells (Dricu et al., 1997). Inhibition of protein glycosylation, perturbation of calcium homeostasis, and reduction of disulfide bonds can provoke accumulation of unfolded protein in endoplasmic reticulum (ER), which is referred to as the 'ER stress' (Schroder and Kaufman, 2005). It has been recently reported that neuronal death in Alzheimer's disease (AD) or ischemia arises from dysfunction of ER (Katayama et al., 2004).

Protein structure and function are greatly influenced by its posttranslational modification, including glycosylation. Previous researches regarding PrP glycosylation were largely restricted in the areas of its cellular localization, protease $\mathrm{K}(\mathrm{PK})$ digestion resistance and protein conversion (Ermonval et al., 2003). In this study, based on the observation of remarkable alterations in the glycosylation ratio of PrP deposits in the brains of the hamsters infected with scrapie $263 \mathrm{~K}$, two $\operatorname{PrP}$ mutants that abolish the glycosylation sites had been generated and introduced into human nueroblastoma cell line SF126. We found that removals of N-linked glycosylation activity on $\operatorname{PrP}$ induced apoptosis phenomena in the cultured cells.

\section{Materials and Methods}

Reagents. Unless otherwise specified, chemical reagents were obtained from Sigma and Merck. Primers were synthesized from BoYa Biotechque Company.

Prion protein in hamster brains purification. Brain homogenates were prepared and prion protein was purified according to procedures described previously (Zhang et al., 2004).

Proteinase $\mathrm{K}$ digestion and western blot analysis of PrP in hamster brains. Proteinase $\mathrm{K}$ digestion and Western blot analysis of PrP were performed as described (Zhang et al., 2004). Quantitative analyses of the immunoblotting signals were carried out using computer-assisted software Image TotalTech.

Recombinant plasmid construction. The full-length human PRNP gene encoding from $1^{\text {st }}$ to $253^{\text {rd }}$ amino acids was amplified by polymerase chain reaction (PCR) from the genome DNA of white blood cells from healthy human, using the following primers: primer HuPrP-F (5'-GGATCCATGGCAAACCTTGGATGCTGG ATG-3' with a BamHI site underlined) and HuPrP-B (5'-GAATTC TTCATCATCATCCCACTATCAGGAA-3' with an EcoRI site underlined). The $762 \mathrm{bp}$-long PCR products were purified and ligated into the vector pGEM-T, generating plasmid pT-HuPrP1253. The human $P R N P$ gene in recombinant plasmid was sequenced with T7 and SP6 primers, revealing the similar nucleotide sequences as PRNP AY569456. To construct human $P R N P$ gene removing the two potential glycosylation sites at the $181^{\text {st }}$ and $197^{\text {th }}$ codons, the PCR and overlapping extension technique were carried out. N181Q mutation was generated with primer m-181-F (5'-TTGTGCACGACTGCGTCCAAATCACAAT CAAGCAGCAC-3') and primer m-181-B (5'-GTGCTGCTTGATT GTGATTTGGACGCAGTCGTGCACAA-3'), using pT-HuPrP1253 as the template. N181Q/N197Q mutation was generated with primer m-197-F (5'-CCACCAAGGGGGAGCAATTCACCGAGA CCGAC-3') and primer m-197-B (5'-GTCGGTCTCGGTGAATTG CTCCCCCTTGGTGG-3'), using human PRNP sequence with N181Q mutation as the template. T183A/T199A mutation was generated with the similar PCR protocol with the primes $\mathrm{m}-183-\mathrm{F}$ (5'-GACTGCGTCAACATCGCAATCAAGCAGCACAC-3'), m-183B (5'-GTGTGCTGCTTGATTGCGATGTTGACGCAGTC-3'), mT199A-F (5'-GGGGAGAACTTCGCCGAGACCGACGT-3') and m-T199A-B (5'-ACGTCGGTCTCGGCGAAGTTCTCCCC-3'). The PCR products were ligated into a PCR-clone vector pMD18-T (TaKaRa, Japan) and verified by sequence analysis. To construct eukaryotic expressing recombinant plasmids, the various inserted $P R N P$ sequences were subcloned into plasmid pcDNA3.1(+)/zeo (Invitrogen, USA) with BamHI and EcoRI, generating the recombinant plasmid $\mathrm{pHuPrP}$ with wild-type human PRNP, plasmids $\mathrm{pHuPrP}-\mathrm{N} 181 \mathrm{Q} / \mathrm{N} 197 \mathrm{Q}$ with mutations at the $181^{\mathrm{st}} / 197^{\text {th }}$ codon and $\mathrm{pHuPrP}-\mathrm{T} 183 \mathrm{~A} / \mathrm{T} 199 \mathrm{~A}$ with mutations at $183^{\text {rd }} / 199^{\text {th }}$ codon.

Cell lines and transient transfection. Human astrocytoma cell line SF126 was maintained in DMEM. Cells were plated into 6well plates one day before transfection. $2 \mu \mathrm{g}$ recombinant plasmids encoding HuPrP, HuPrP N181Q/N197Q or HuPrP T183A/T199A, as well as the vector pcDNA3.1, were transfected into the monolayer cells with Lipofectamine 2000 transfection reagent (Invitrogen, USA). Cells were harvested at $30 \mathrm{~h}$ after transfection.

Cell sample preparation and western blot analysis. Cells were lysed in the solution containing $150 \mathrm{mM} \mathrm{NaCl}, 50 \mathrm{mM}$ Tris- $\mathrm{HCl}$ (pH 7.5), 0.5\% Triton X-100, 0.5\% NP-40, and 0.5\% sodium deoxycholate, supplemented with protease inhibitors $(1 \mu \mathrm{g} / \mathrm{ml}$ pepstatin and leupeptin, $1 \mathrm{mM}$ phenylmethylsulfonyl fluoride, 2 $\mathrm{mM}$ EDTA). Lysates were centrifuged for $20 \mathrm{~min}$ at $14,000 \mathrm{~g}$ to remove debris. The supernatants were collected and stored at $-70^{\circ} \mathrm{C}$ (Metrailler-Ruchonnet et al., 2007 and Yu et al., 2005).

Samples were subjected to $15 \%$ sodium dodecyl sulfate polyacrylamide gel electrophoresis (SDS-PAGE), and subsequently transferred to nitrocellulose membranes. The primary antibodies used in Western blot assays included monoclonal antibodies 3F4 (DAKO), Bcl-2 (DAKO), Bax and beta-actin (Cell Signal Technology). The secondary antibody was horseradish peroxidaselabeled goat anti-mouse (Santa Cruz). Detection of signal was performed with an enhanced chemiluminescence detection kit (Amersham-Pharmacia Biotech).

Peptide N-glycosidase F (PNGase F) digestion. Cell lysates were treated with PNGase F (250 units/ml, New England Biology, Inc) for $16 \mathrm{~h}$ at $37^{\circ} \mathrm{C}$. PrP signals in the preparations were analyzed by $15 \%$ SDS-PAGE and PrP specific Western blots.

Immunofluorescence assay. SF126 cells, which were grown on the glass slides, were fixed with cold $4 \%$ paraformaldehyde solution for $10 \mathrm{~min}$, and blocked with $3 \%$ bovine serum albumin (BSA) for $30 \mathrm{~min}$ at $37^{\circ} \mathrm{C}$. Cells were incubated with the primary 
antibodies 3F4 (1: 50 in PBS containing 2\% BSA) at $37^{\circ} \mathrm{C}$ for 90 min and subsequently incubated with FITC labeled secondary antibody $(1: 200 \mathrm{in}$ PBS $)$ at $37^{\circ} \mathrm{C}$ for $30 \mathrm{~min}$. Cells were examined with a confocal microscope (Zeiss). The images were captured using LSM Software.

Nuclei morphological analysis. Cells were stained with 4', 6diamino-2-phenylindole dihydrochloride (DAPI, Sigma). Briefly, cells were fixed for $20 \mathrm{~min}$ in fresh $4 \%$ paraformaldehyde, rinsed with PBS, and stained for 15 min with $1 \mu \mathrm{M}$ DAPI in PBS at $37^{\circ} \mathrm{C}$. After being carefully washed twice, chromatin condensation or nuclei smash were observed with fluorescence microscopy (330$380 \mathrm{~nm}$ excitations) and the cells with morphological abnormality were counted in the context of 500 cells randomly.

Apoptosis rate assay by Annexin/PI labeling. $30 \mathrm{~h}$ after transfection, cells were harvested. $0.5 \times 10^{6}$ cells of each preparation were duallabeled with annexin V and propidium iodide (PI) with Annexin VFITC Apoptosis Detection kit (BD) according to the manufacturer's instructions, and subjected to a flow cytometry analyzer (BD).

\section{Measurement of mitochondrial membrane permeability changes.} Cellular mitochondrial membrane permeability changes $(\Delta \Psi \mathrm{m})$ were determined by staining of rhodamine 123 . After transfected for $30 \mathrm{~h}, \mathrm{SF} 126$ cells were resuspended in PBS at a density of $1 \times 10^{6} \mathrm{cells} / \mathrm{ml}$. Rhodamine 123 was added to each preparation at a final concentration $(10 \mu \mathrm{g} / \mathrm{ml})$ for $60 \mathrm{~min}$. Cytofluorometric analysis was carried out using a flow cytometry analyzer (BD).

Statistics. All the metric data were given as means \pm SD. All the enumerative data were given as percentages. For statistical comparison, $t$ test and $u$ test were used. $P$ values smaller than 0.05 were considered to be statistically significant.

\section{Results}

Abnormal glycosylation patterns of total PrP were observed in brains of hamsters infected with scrapie. Scanning of PrP signals in western blots revealed that glycosylation patterns and ratios of $\operatorname{PrP}$ in the brain tissues of the hamsters infected with scrapie agent $263 \mathrm{~K}$ were variable during the clinical course. The di-glycosylation form of PrP was predominant in all six healthy animals, occupying $81.07 \pm 7.09 \%$ of total contents of PrP, while the monoglycosylation and non-glycosylation took only $13.37 \pm 5.92 \%$ and $5.55 \pm 1.59 \%$, respectively (Table 1 ). Contrast to the

Table 1. Comparison of each glycosylated PrP form in brains of normal hamsters and the hamsters infected with scrapie strain $263 \mathrm{~K}$

\begin{tabular}{lcc}
\hline \multicolumn{1}{c}{ Content } & $\begin{array}{c}\text { Control group } \\
(\mathrm{N}=6)(\%)\end{array}$ & $\begin{array}{c}\text { Infected group } \\
(\mathrm{N}=15)(\%)\end{array}$ \\
\hline Di-glycosylated PrP & $81.07 \pm 7.09$ & $41.01 \pm 8.88$ \\
Mono-glycosylated PrP & $13.37 \pm 5.92$ & $37.09 \pm 13.05$ \\
Non-glycosylated PrP & $5.55 \pm 1.59$ & $21.91 \pm 11.24$ \\
\hline
\end{tabular}

normal control, the ratios of di-glycosylation $\operatorname{PrP}$ of the infected hamsters declined $(41.01 \pm 8.88 \%, P<0.01)$, while that of non-glycosylation PrP increased in the infected groups $(21.91 \pm 11.24 \%, P<0.01$, Table 1). These data indicates the increases of non-glycosylation $\operatorname{PrP}$ are the common feature in the terminal course of the scrapie-infected experimental animals.

Exchanges of nucleotides within the glycosylation sites in PRNP gene abolished the glycosylation of the expressed PrP. Two full-length PrP mutants (from amino acid $1^{\text {st }}$ to $253^{\text {rd }}$ ), N181Q/N197Q and T183A/T199A, were constructed, in which site-directed mutations were employed into the two glycosylation sites at the positions of codon 181 and 197, as well as codon 183 and 199, respectively. Transient transfection of p-HuPrP expressing wild type human PrP in SF126 cells induced a brain tissues-like PrP expression manner, that the expressed PrP signals occupied the positions from 27 to 36 $\mathrm{kDa}$ with several distinct bands in Western blot (Fig. 1A). Treatment with PNGase F resulted in a single $27 \mathrm{kDa}$ band, demonstrating the expressed PrP contained fully or partially glycosyl forms (Fig. 1A). In contrast, transfection of the recombinant plasmids either $\mathrm{pHuPrP}-\mathrm{N} 181 \mathrm{Q} / \mathrm{N} 197 \mathrm{Q}$ or $\mathrm{pHuPrP}-$ T183A/T199A demonstrated only a signal $27 \mathrm{kDa}$ PrP band, while treatment of PNGase F did not influence the mobility position of the expressed PrPs (Fig. 1A), implying that the expressed PrP proteins in the cells transfected with the two

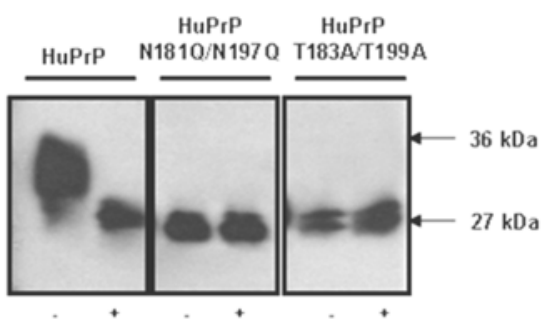

(B)

PNGase F

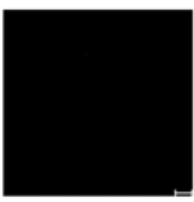

pcDNA3.1

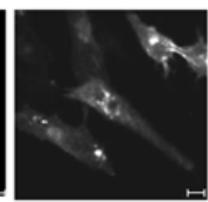

HuPrP

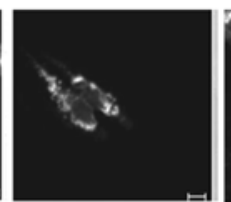

HuPrP N181Q/N197Q

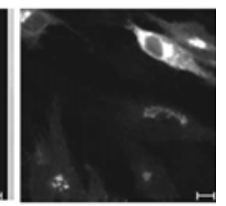

HuPrP T183A/T199A
Fig. 1. (A) Western blot assays for expressions of wild type and mutated PrP proteins in SF126 cells. Cell lyses were prepared 30 $\mathrm{h}$ after transfection and protein concentrations in each preparation were equilibrated before SDS-PAGE. PrP specific signals were detected with monoclonal antibody $3 \mathrm{~F} 4$ and horseradish peroxidase-labeled goat anti-mouse described elsewhere. Individual PrP proteins are shown above. Treatments of PNGase $\mathrm{F}$ are shown in the bottom, +: with PNGase F, -: without PNGase F. Molecular mass markers are indicated to the right. (B) Immunofluorescence assays for expressions of wild type and mutated PrP proteins in SF126 cells. PrP specific signals were detected with monoclonal antibody $3 \mathrm{~F} 4$ and FITC-labeled goat anti-mouse $\operatorname{IgG}(5 \mu \mathrm{m})$. 


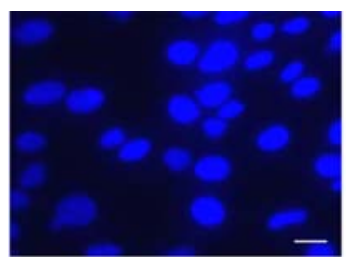

cell

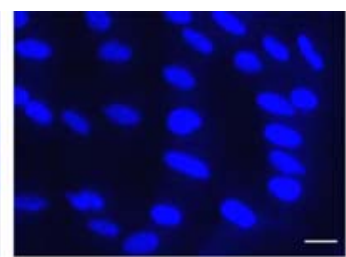

PcDNA

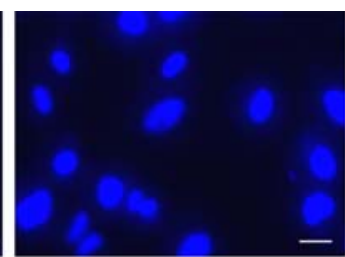

HuPrP

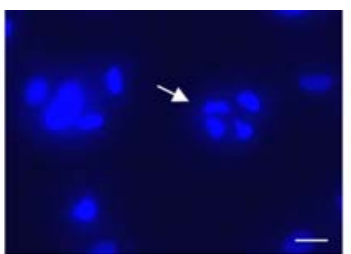

HuPrP N181Q/N197Q

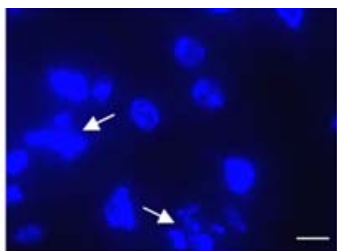

HuPrP T183AЛT199A

Fig. 2. Cellular nuclei morphological Changes of the cells $30 \mathrm{~h}$ after transfected with recombinant plasmids p-HuPrP, p-HuPrP-N181Q/ N197Q and p-HuPrP-T183A/T199A as well as mock plasmid. Cellular nuclei abnormalities including nuclei shrunken, denseness and nuclei broken to pieces are shown with white arrows $(5 \mu \mathrm{m})$.

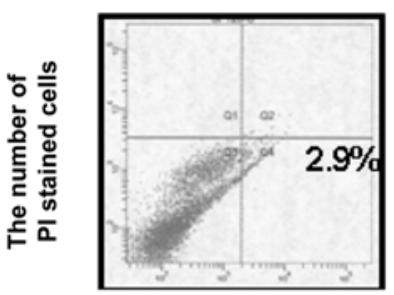

cell
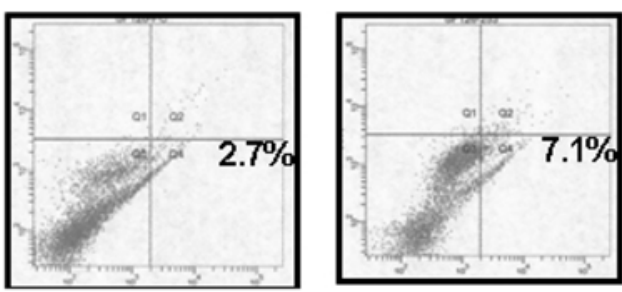

The number of Annexin V-FITC stained cells pcDNA3.1
HuPrP

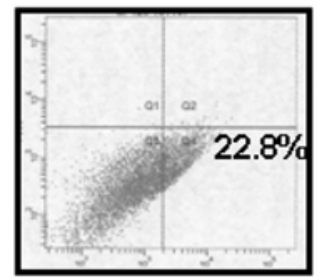

HuPrP N181Q/N197Q

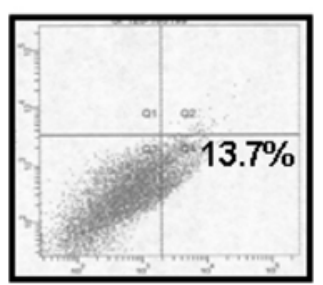

HuPrP T183AT199A

Fig. 3. Annexin V/PI double staining assays of SF126 $30 \mathrm{~h}$ after transfected with recombinant plasmids p-HuPrP, p-HuPrP-N181Q/ N197Q and p-HuPrP-T183A/T199A as well as mock plasmid. X axis indicates the numbers of Annexin V-FITC stained cells. Y axis indicates the numbers of PI strained cells. The percentages of the cells labeled with only Annexin V (counted in Q4 region) in each preparation are shown in each figure.

PrP mutants were non-N-glycosylated forms.

Immunofluorescent staining of the cells receiving various PrP expressing plasmids revealed a similar pattern of intracellular localization that the expressions of PrPs mainly located in cytoplasma (Fig. 1B). Analyses of the intensity of fluorescence of the cells expressing wild-type HuPrP, mutant PrP N181Q/N197Q and HuPrP T183A/T199A did not show any significant difference.

\footnotetext{
Removal of glycosylation sites within PrP induced apoptosis in the transiently transfected cells. To see the possible effects of exogenous PrP or PrP mutants on the growth activities of the transfected cells, the recombinant PrP plasmids p-HuPrP, p-HuPrP-N181Q/N197Q and p-HuPrP-T183A/T199A were transiently transfected into SF126 individually, while plasmid pcDNA3.1 and normal cell were employed as the plasmid and negative controls in parallel. $30 \mathrm{~h}$ after transfection, cells were monitored and harvested for further studies. Morphological observations with DAPI staining revealed cellular nuclei abnormalities including nuclei shrunken, denseness and nuclei broken to pieces in the preparations of p-HuPrP-N181Q/N197Q and p-HuPrP-T183A/T199A, whereas few morphological alteration has been found in the control preparations (Fig. 2). Counting randomly 500 cells each preparation showed that the morphological abnormalities were more easily identified and remarkably severe in the transfections of p-HuPrPN181Q/N197Q (78/500 cells) and p-HuPrP-T183A/T199A
}

(90/500 cells) than that of wild-type p-HuPrP (16/500 cells), with significant statistical difference $(P<0.01$, u test).

To get more evidences of apoptosis, the various transfected cells were subjected into a flow cytometry analyzer for counting the annexin/PI labeled cells. Comparison with the SF126 cells transfected with plasmid pcDNA3.1 and normal cell, transfection with $\mathrm{p}-\mathrm{HuPrP}$ induced more cells $(7.1 \%)$ to be labeled only with annexin $\mathrm{V}$ (the cell counted in Q4 region) (Fig. 3). Corresponding well to what has been found in DAPI staining, HuPrP-N181Q/N197Q induced more cell apoptosis $(22.8 \%)$, showing significant statistics differences $(P<0.01, u$ test) compared with that of HuPrP (Fig. 3). However, HuPrP-T183A/T199A induced only limitedly increase of apoptosis in SF126 cells (13.7\%) (Fig. 3).

Cells receiving various recombinant PrP plasmids were stained with a commercial TUNEL kit at $30 \mathrm{~h}$ post-transfection, in order to see the signals of terminal apoptosis. Almost all mock cells and the cells transfected with plasmid pcDNA3.1 were TUNEL-staining negative, showing green color after restaining of methyl green (Fig. 4). A few dark brown-stained nuclei were observed in the preparation of wild type PrP (Fig. 4), whereas remarkably more positive stained nuclei and particles were distributed in the preparations of PrP mutants HuPrP-N181Q/N197Q and HuPrP-T183A/T199A (Fig. 4). No significant differences in the distribution and intensity of positive signals were identified between the two mutants. 


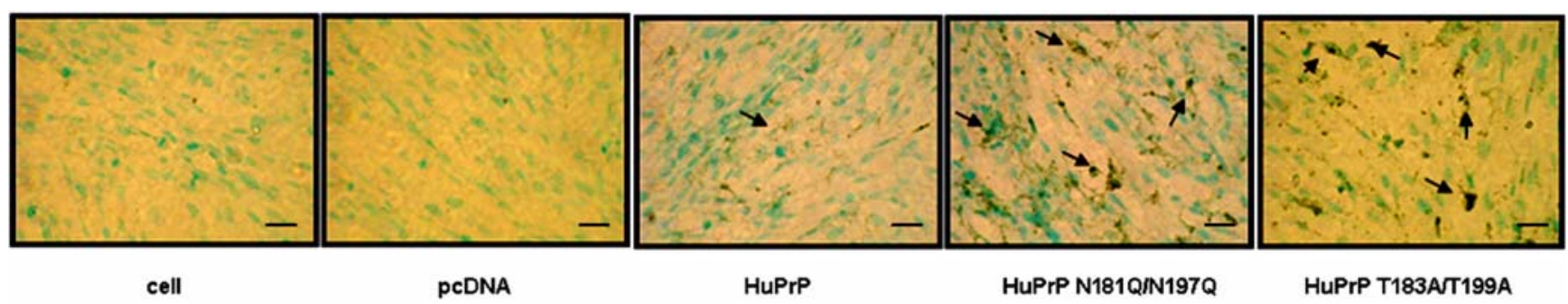

Fig. 4. TUNEL staining assays of SF126 cells $30 \mathrm{~h}$ after transfected with recombinant plasmids p-HuPrP, p-HuPrP-N181Q/N197Q and p-HuPrP-T183A/T199A as well as mock plasmid. The dark brown-stained cells were shown with black arrows $(25 \mu \mathrm{m})$.

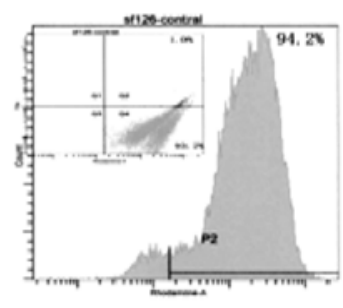

cell

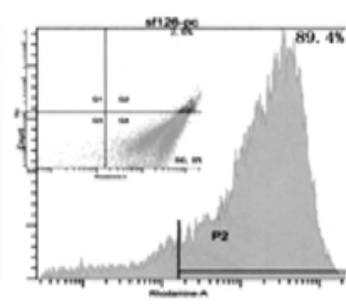

pcDNA3.1

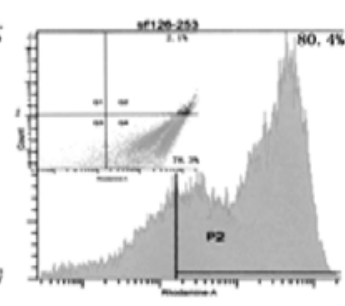

HuPrP

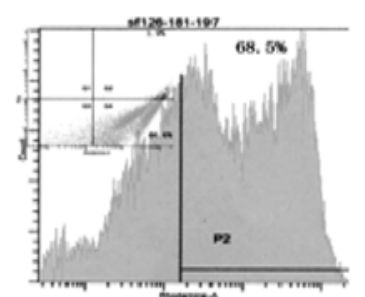

HuPrP

N181Q/N197Q

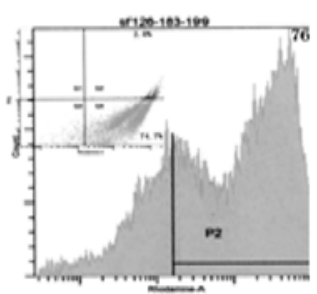

HuPrP T183AT199A

Fig. 5. Rhodamine 123 staining assays of SF126 cells $30 \mathrm{~h}$ after transfected with recombinant plasmids p-HuPrP, p-HuPrP-N181Q/ N197Q and p-HuPrP-T183A/T199A as well as mock plasmid.

\begin{abstract}
Removal of glycosylation sites within PrP reduced the mitochondrial membrane potential in the transiently transfected cells. To determine whether the cellular mitochondrial membrane potential was influenced by transfection of PrP, SF126 cells receiving different glycosylated forms of PrPs were treated with rhodamine 123 and $\Delta \Psi \mathrm{m}$ was assessed by flow cytometric analysis. Comparing with the $\Delta \Psi \mathrm{m}$ of the cells transfected with plasmid pcDNA3.1 control, transient expression of $\mathrm{HuPrP}$ induced $80.4 \%$ decreased $\Delta \Psi \mathrm{m}$, while expressions of HuPrP-N181Q/N197Q and HuPrP-T183A/ T199A caused $68.5 \%$ and $76.7 \%$ decreased, respectively (Fig. $5)$.
\end{abstract}

The Bcl-2 and Bax involved in the apoptosis courses inducted by non-N-glycosylated PrP. To test the potential alterations of apoptosis associated protein, the levels of Bcl-2 and Bax in the individual preparations were evaluated with Western blots. Compared with the control preparations, the amounts of Bcl-2 were similar in SF126 cells transfected with p-HuPrP, whereas SF126 cells receiving p-HuPrP-N181Q/ N197Q or p-HuPrP-T183A/T199A showed remarkably lower amounts of Bcl-2 (Fig. 6A). Quantitative evaluations of the gray values of the individual immunoblot signal after scanning identified that the Bcl-2 relative gray values of the preparations of N181Q/N197Q and T183A/T199A reduced to $39.4 \%$ and $36.1 \%$ of that of the mock cells (Fig. 6B). In contrast, Western blots showed that Bax of the cells receiving p-HuPrP-N181Q/N197Q and p-HuPrP-T183A/T199A were obviously increased, while the cells transfected with wild-type HuPrP maintained almost unchanged (Fig. 6A). Compared with that of the mock cells, the gray values of Bax of the preparations with $\mathrm{N} 181 \mathrm{Q} / \mathrm{N} 197 \mathrm{Q}$ and $\mathrm{T} 183 \mathrm{~A} / \mathrm{T} 199 \mathrm{~A}$ raised to $147.9 \%$ and $136.1 \%$, respectively (Fig. $6 \mathrm{~B}$ ). Calculating the ratios of $\mathrm{Bax} / \mathrm{Bcl}-2$ amounts of individual preparations proposed roughly 4 folds increased in the cells receiving the mutated HuPrP compared with that of the mock, while unchanged in the cells receiving the wild-type HuPrP (Fig. $6 \mathrm{~B})$. These results indicated that Bcl-2 and Bax possibly involved in the apoptosis processes of the transfected cells triggered by the exogenous mutated PrP.

\section{Discussion}

In this work, we start our study from the observations of the changes of PrP glycosylation ratios in the brain tissues of the hamsters infected with scrapie $263 \mathrm{~K}$. Apoptosis phenomenon has been described in naturally occurred scrapie sheep, CJD cases and the experimental bioassays in mice infected with different TSE agents, as well as in some cultured cell lines infected with TSE agents in vitro (Cronier et al., 2004). This report and our previous study (Gao et al., 2004) propose the time-related histopathological appearance of apoptosis in the brain tissues of the hamsters infected with scrapie strain 263K, which correlates well with the deposits of $\operatorname{PrP}^{\mathrm{Sc}}$. In addition to the remarkable increases of absolute amounts of all three PrP isoforms at the terminal courses of experimental scrapie in hamsters, a significant ratio alteration of the three isoforms has been observed, that the di-glycosylation $\operatorname{PrP}$ decreases, while the mono-glycosylation $\operatorname{PrP}$ and non- 
(A)

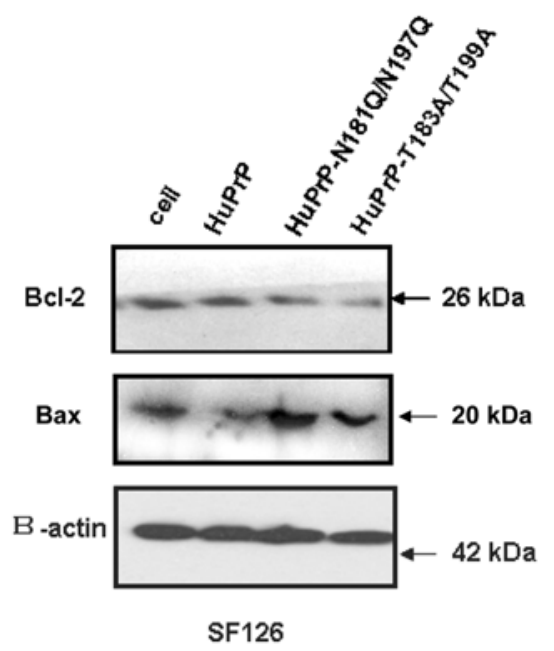

(B)

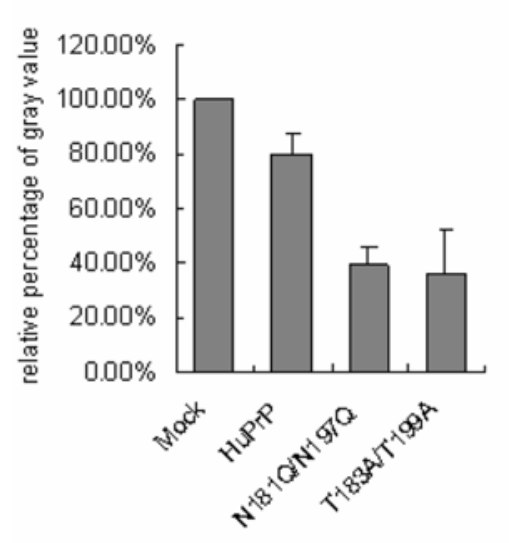

Bax

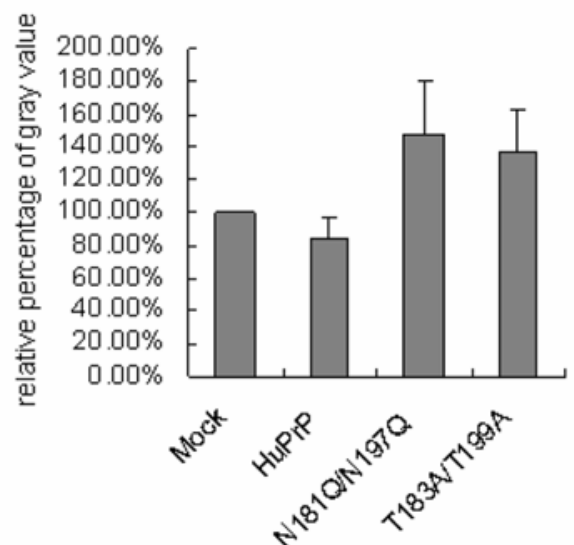

BaxBcl-2

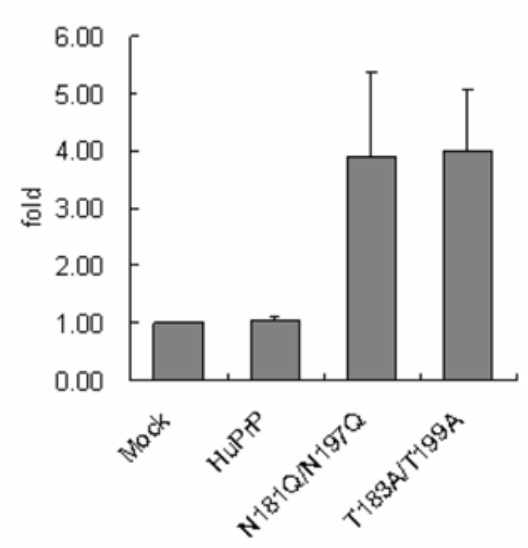

Fig. 6. Western blot assays for the presences of Bcl-2 and Bax in SF126 cells transfected with recombinant plasmids p-HuPrP, pHuPrP-N181Q/N197Q and p-HuPrP-T183A/T199A as well as mock plasmid. A. Western blot. Cell lyses are prepared $30 \mathrm{~h}$ after transfection and protein concentrations in each preparation are equilibrated before SDS-PAGE. The expressions of beta-actin were used as internal control. Molecular mass markers are indicated to the right. B. Quantitative analyses of the Bcl-2 and Bax immunoblots in each preparation. The average gray value of $\mathrm{Bcl}-2$ or Bax was obtained from three independent tests. The changes of $\mathrm{Bcl}-2$ or $\mathrm{Bax}$ in each preparation were indicated as the relative percentage of gray value obtained from dividing the gray value of the immnuoblot of individual test by that of the mock. The left, middle and right panels illustrated changes of $\mathrm{Bcl}-2$, Bax and the ratios of Bax/Bcl-2 in each test, respectively.

glycosylation PrP increase. Well accordance of the appearance of apoptosis with the alteration of PrP glycosylation ratios in scrapie-infected animals implies potential differences of three isoforms of PrP molecules in the effect on cell life cycle.

Both PrP mutants N181Q/N197Q and T183A/T199A are able to abolish the attachment of N-linked glycan on exogenously expressed PrP molecules in SF126 cells. Expression of two mutants induced similar cell apoptosis patterns, except in Annexin/PI labeling assay. Carefully analyses the expressed PrP proteins in tested cell lines with FITC-immunostaining showed similar intracellular morphological distributions in SF126. In line with the works from Lehmann et al. (Lehmann and Harris, 1997) in transient transfections, the expressed PrP proteins in our experiment orientating mostly at cytoplasm without clear evidences on the surface of cellular membrane. The slight differences of the two mutants in Annexin /PI labeling assay may not be the result of different cellular trafficking and distribution. Similar protein expressing, processing and distributing features of identical mouse PrP glycosylation mutants have also been seen in different tissuederived cell lines, including neuroblastoma cells, fibroblast cells and ovarian cells (Neuendorf et al., 2004).

Anti-apoptotic members of the Bcl-2 family of proteins, such as Bcl-2, are located in the outer mitochondrial membrane and act to promote cell survival. We demonstrate that the $\operatorname{PrP}$ glycosylation mutants, but not the wild-type PrP, decrease the quantity of Bcl-2 and increase the quantity of Bax in the transiently transfected cells, which is well correlated with the 
changes of the cellular mitochondrial membrane potential after expressions of aglycosal PrPs. It indicated that the change of mitochondria function might be take part in the apoptosis. Introduction of Bcl-2 into the PrP-null neural cells can attenuate the sensitivity to serum deprivation (Kuwahara et al., 1999). Contrast to the wild-type PrP, PrP106-126 fragment decreases the level of Bcl-2 mRNA in neuron cells in vitro experiments (Perovic et al., 1997). PrP mutants, e.g. deletion of four octapeptide repeats of PrP FFI-related mutant D178N and familial CJD-related mutant T183A, completely or partially eliminate the neuroprotective effect of $\operatorname{PrP}$ (Bounhar et al., 2001, Roucou and LeBlanc, 2005). Expressions of Bcl2 mRNA and protein are significantly decreased in the brains of $263 \mathrm{~K}$ scrapie-infected hamsters (Park et al., 2000). These data demonstrate that structural or conformational changes of PrP proteins, including removal of PrP glycosylation process, will break the Bcl-2/Bax network, resulting in influences in cell life. In fact, changes of protein glycosylation processes leading in cells apoptosis possibly via Bcl-2 family members had been described elsewhere (Niederer et al., 2005 and Reimertz et al., 2003).

Expressions of PrP mutants whose two glycosylation sites have been demolished in the cultured cells propose firstly the evidence that non-glycosylated PrP protein possibly provokes cell apoptosis. Inhibition of N-linked glycosylation processes with tunicamycin induces apoptosis effect on the cultured cells (Dricu et al., 1997). As a glycoprotein, PrP undergoes post-translational modification through ER and Golgi body, in which $\operatorname{PrP}$ is glycosylated, and subsequently locates at cellular membrane. Immature PrP, possibly including aglycosal PrP, is believed being degraded through cellular proteolysis. Although the physiological meaning of glycosylation on PrP is far from clear, some previous studies reveal that N-linked glycan can help the folding process of newly synthesized protein (Ermonval et al., 2003). Glycosylation modification is able to protect the surface structures of $\operatorname{PrP}^{\mathrm{C}}$, that the free energy for conversion of the diglycosal $\operatorname{PrP}^{\mathrm{C}}$ to $\operatorname{PrP}^{\mathrm{Sc}}$ is probably higher resulting in less efficient in conformational conversion (Zuegg and Gready, 2000). The transgenic mice bearing glycosylation deficiency at either one of the two glycan attachment sites of $\operatorname{PrP}^{\mathrm{C}}$ show more sensitivity to the infections of BSE and scrapie agents (Neuendorf et al., 2004). These data strongly highlight that removal of N-linked glycan on PrP molecules bring about or stress cell damage, possibly through cell apoptosis process.

Acknowledgments We thank Dr. Jin Zhang for excellent technique assistance and animal bioassays. We are also grateful to Dr. Xu Tang for supporting health human genome DNA. This work was supported by Chinese National Natural Science Foundation Grants 30130070, 30070038 and 39928018, National High-tech Development Project (863 Project) 2001AA215391, and EU Project QLRT 200001441.

\section{References}

Bate C. and Williams A. (2004) Role of glycosylphosphatidylinositols in the activation of phospholipase A2 and the neurotoxicity of prions. J. Gen. Virol. 85, 3797-3804.

Bounhar Y., Zhang Y. and Goodyer C. G. (2001) Prion protein protects human neurons against Bax-mediated apoptosis. $J$. Biol. Chem. 276, 39145-39149.

Chabry J., Ratsimanohatra C., Sponne I., Elena P. P., Vincent J. P. and Pillot T. (2003) In vivo and in vitro neurotoxicity of the human prion protein $(\mathrm{PrP})$ fragment P118-135 independently of PrP expression. J. Neurosci. 23, 462-469.

Ciesielski-Treska J., Grant N. J., Ulrich G., Corrotte M., Bailly Y., Haeberle A. M., Chasserot-Golaz S. and Bader M. F. (2004) Fibrillar prion peptide (106-126) and scrapie prion protein hamper phagocytosis in microglia. Glia. 46, 101-115.

Cronier S., Laude H. and Peyrin J. M. (2004) Prions can infect primary cultured neurons and astrocytes and promote neuronal cell death. Proc. Natl. Acad. Sci. USA 101, 12271-12276.

Dricu A., Carlberg M., Wang M. and Larsson O. (1997) Inhibition of N-linked glycosylation using tunicamycin causes cell death in malignant cells: role of down-regulation of the insulin-like growth factor 1 receptor in induction of apoptosis. Cancer Res. 57, 543-548.

Ermonval, M., Mouillet-Richard, S., Codogno, P., Kellermann, O. and Botti, J. (2003) Evolving views in prion glycosylation: functional and pathological implications. Biochimie. 85, 33-45.

Gao, J. M., Gao, C., Han, J., Zhou, X. B., Xiao, X. L., Zhang, J., Chen, L., Zhang B. Y., Hong, T. and Dong, X. P. (2004) Dynamic analyses of $\operatorname{PrP}$ and $\operatorname{PrP}(\mathrm{Sc})$ in brain tissues of golden hamsters infected with scrapie strain $263 \mathrm{~K}$ revealed various PrP forms. Biomed. Environ. Sci. 17, 8-20.

Katayama, T., Imaizumi, K., Manabe, T., Hitomi, J., Kudo, T. and Tohyama, M. (2004) Induction of neuronal death by ER stress in Alzheimer's disease. J. Chem. Neuroanat. 28, 67-78.

Kuwahara, C., Takeuchi, A. M., Nishimura, T., Haraguchi, K., Kubosaki, A., Matsumoto, Y., Saeki, K., Matsumoto, Y., Yokoyama, T., Itohara, S. and Onodera, T. (1999) Prions prevent neuronal cell-line death. Nature 400, 225-226.

Lehmann, S. and Harris, D. A. (1997) Blockade of glycosylation promotes acquisition of scrapie-like properties by the prion protein in cultured cells. J. Biol. Chem., 272, 21479-21487.

Metrailler-Ruchonnet, I., Pagano, A., Carnesecchi, S., Ody, C., Donati, Y. and Barazzone, A. C. (2007) Bcl-2 protects against hyperoxia-induced apoptosis through inhibition of the mitochondria-dependent pathway. Free Radic. Biol. Med. 42, 1062-1074.

Neuendorf, E., Weber, A., Saalmueller, A., Schatzl, H., Reifenberg, K., Pfaff, E. and Groschup, M. H. (2004) Glycosylation deficiency at either one of the two glycan attachment sites of cellular prion protein preserves susceptibility to bovine spongiform encephalopathy and scrapie infections. J. Biol. Chem. 279, 53306-53316.

Niederer, K. E., Morrow, D. K., Gettings, J. L., Irick, M., Krawiecki, A. and Brewster, J. L. (2005) Cypermethrin blocks a mitochondria-dependent apoptotic signal initiated by deficient N-linked glycosylation within the endoplasmic reticulum. Cell Signal. 17, 177-186. 
Nishimura, T., Sakudo, A., Nakamura, I., Lee, D. C., Taniuchi, Y., Saeki, K., Matsumoto, Y., Ogawa, M., Sakaguchi, S., Itohara, S. and Onodera, T. (2004) Cellular prion protein regulates intracellular hydrogen peroxide level and prevents copperinduced apoptosis. Biochem. Biophys. Res. Commun. 323, 218222.

Park, S. K., Choi, S. I., Jin, J. K., Choi, E. K., Kim, J. I., Carp, R. I. and Kim, Y. S. (2000) Differential expression of Bax and $\mathrm{Bcl}-2$ in the brains of hamsters infected with $263 \mathrm{~K}$ scrapie agent. Neuroreport. 11, 1677-1682.

Perovic, S., Schroder, H. C., Pergande, G., Ushijima, H. and Muller, W. E. (1997) Effect of flupirtine on Bcl-2 and glutathione level in neuronal cells treated in vitro with the prion protein fragment (PrP106-126). Exp. Neurol. 147, 518524.

Prusiner, S. B. (1998) Prions. Proc. Natl. Acad. Sci. USA 95, 13363-13383.

Roucou, X. and LeBlanc, A. C. (2005) Cellular prion protein neuroprotective function: implications in prion diseases. $J$. Mol. Med. 83, 3-11.

Schroder, M. and Kaufman, R. J. (2005) ER stress and the unfolded protein response. Mutat. Res. 569, 29-63.

Zhang, J., Chen, L., Zhang, B. Y., Han, J., Xiao, X. L., Tian, H. Y., Li, B. L., Gao, C., Gao, J. M., Zhou, X. B., Ma, G. P., Liu, Y., Xu, C. M. and Dong, X. P. (2004) Comparison study on clinical and neuropathological characteristics of hamsters inoculated with scrapie strain $263 \mathrm{~K}$ in different challenging pathways. Biomed. Environ. Sci. 17, 65-78.

Yu, L. C., Flynn, A. N., Turner, J. R., Buret A. G. (2005) SGLT-1mediated glucose uptake protects intestinal epithelial cells against LPS-induced apoptosis and barrier defects: a novel cellular rescue mechanism? FASEB J. 19, 1822-1835.

Zuegg, J. and Gready, J. E. (2000) Molecular dynamics simulation of human prion protein including both N-linked oligosaccharides and the GPI anchor. Glycobiology. 10, 959-974. 